etwas niedriger aus als die der Elektrophorese. Da für die Elektrophorese das Beersche Gesetz bei der Transparenzphotometrie nicht streng erfüllt ist $(11,12,6$, $16,13,8)$, ist diese Differenz erklärlich. - Hämolytische Seren, auch solche mit leichten Hämolysegraden, sind für die TES-Äthanol-Methode wie für die Elektro- phorese $(14,15)$ ungeeignet. So ist man mit dem TESÄthanol-Verfahren in der Lage, schnell und exakt den Albumingehalt des Serums festzustellen und durch Subtrahieren dieses Wertes vom Gesamteiweiß des Serums den Globulinanteil und damit das Verhältnis Albumin zu Globulin zu ermitteln.

\title{
Literatur
}

1. Pillemer, L. und M. C. Hutchinson, J. biol. Chemistry 158 , 299 (1945). - 2. Lavine, S., Arch. Biochem. Biophysics 50, 515 (1954). - 3. Delaville, M., G. Delaville und J. Delavirle, Année pharmaceut. Paris, 12, 109 (1954). - 4. Schwert, G. W., J. Amer. chem. Soc. 79, 139 (1957). - 5. KorNER, A. und J. R. Debro, Nature (London) 178, 1067 (1956). - 6. Owen, J. A., Analyst, 81, 26 (1956). - 7. MrChAEL, S. E., IV. Intern. Biochem. Kongreß, Wien (1958). - 8. WALSCH, J. R., F. L. Humoller und A. L. DunN, J. Laborat. Clin. Med., S. Louis 46, 772 (1955). 9. Kallee, E., F. Lohss und W. OppermanN, Z. Naturforsch.
Teil B, 12, 777 (1957). - 10. Weichselbaum, T. E., Amer. J. Clin. Path. (Techn. Sect.) 10, 40 (1946). - 11. Fuchs, W. und A. FlACH, Klin. Wschr. 33, 903 (1955). - 12. Osterhuits, H. K., J. Laborat. Clin. Med., S. Louis 44, 280 (1956). - 13. Schulz, D. M. und M. Holdcraft, Amer. J. Clin. Path. 26, 215 (1956). - 14. SANDKüHLER, S., Dtsch. med. J. 13, 266 (1962). - 15. SCHOEN, R. und $\mathrm{H}$. SüDHOF, Biochemische Befunde in der Differentialdiagnose innerer Krankheiten. Georg Thieme Verlag, Stuttgart (1962). 16. Hinsberg, K. und K. Lang, Medizinische Chemie. 3. Auflage S. 996, Urban \& Schwarzenberg, München, Berlin (19.57).

Dr. med. Z. F. Ch. Kachani $23 \mathrm{Kiel}$

Brunswiker Str. 2-6

\section{Sauerstoff-Aufnahme von Humanplasma nach Zugabe von Kobalt}

\author{
Von \\ J. Dittmann und P. Sachtreben \\ Aus der Universitäts-Kinderklinik Homburg, Saar, und der Landesklinik Neunkirchen-Koblhof, Saar \\ (Direktor: Prof. J. B. Mayer)
}

(Der Schriftleitung zugegangen am 7. Februar 1964)

\begin{abstract}
Gibt man $6 \mu \mathrm{Mol} \mathrm{CoSO}{ }_{4}$ gelöst in $0,2 \mathrm{~m} l$ Wasser unter $\mathrm{O}_{2}$ bei $38^{\circ} \mathrm{zu} 0,6 \mathrm{~m} l$ Plasma, so wird die $\mathrm{O}_{2}$-Aufnahme des Plasmas stark stimuliert. Gibt man die gleiche Menge CoSO ${ }_{4}$ zu der entsprechenden Menge, nämlich $1 \mathrm{ml}$, Blut, so bleibt der beschriebene Effekt aus.

The addition of $6 \mu \mathrm{mol}$. of $\mathrm{CoSO}_{4}$ in $0.2 \mathrm{ml}$. of water to $0.6 \mathrm{~m} /$. of plasma under $\mathrm{O}_{2}$ at $38^{\circ}$ causes a marked stimulation of the $\mathrm{O}_{2}$-uptake of the plasma. If the same amount is added to a corresponding amount of blood $(1 \mathrm{ml}$.), there is no effect.
\end{abstract}

Wir haben die Sauerstoff-Aufnahme von Blutzellen in Gegenwart verschiedener Kobalt-II-Ionen-Konzentrationen manometrisch gemessen und dabei folgende Beobachtung gemacht: Gibt man $6 \mu \mathrm{Mol} \mathrm{Co}^{2+}$ gelöst in $0,2 \mathrm{~m} l$ Wasser zu $1 \mathrm{~m} l$ Blut, so wird die folgende $\mathrm{O}_{2}-$ Aufnahme gegenüber den Kontrollen ohne $\mathrm{Co}^{2+}$-Zusatz etwas erhöht; gibt man die Lösung von $6 \mu \mathrm{Mol} \mathrm{Co}^{2+}$ in $0,2 \mathrm{~m} l$ Wasser jedoch zu der entsprechenden Menge, nämlich $0,6 \mathrm{~m} l$ Plasma, so setzt nach einer gewissen Inkubationszeit eine sehr kräftige $\mathrm{O}_{2}$-Aufnahme ein.

\section{Methode}

Blut wurde aus der Armvene in eine mit Heparin benetzte Spritze gezogen und anschließend sofort in vorbereitete Kegelgefäße von etwa $15 \mathrm{~m} l$ Inhalt pipettiert. Für die Versuche mit Plasma wurde das Blut unmittelbar nach der Entnahme $10 \mathrm{Min}$. lang bei etwa
$3000 \mathrm{~g}$ zentrifugiert. Genau $30 \mathrm{Min}$. nach Blutentnahme wurden die Gefäße in den Thermostaten gehängt und anschließend $5 \mathrm{Min}$. ohne Schütteln bei $38^{\circ}$ mit reinem $\mathrm{O}_{2}$ durchströmt. $50 \mathrm{Min}$. nach Entnahme wurde die Schüttelung der Gefäße begonnen: Warburg. Apparat Typ S 85 der Fa. Braun, 100 Schüttelungen pro Min., Amplitude $2 \mathrm{~cm}$. Genau $60 \mathrm{Min}$. nach Blutentnahme erfolgte die erste Manometer-Ablesung, genau $90 \mathrm{Min}$. nach Blutentnahme wurde aus dem Seitenanhang $\mathrm{Co}^{2+}$ zugegeben. In der graphischen Darstellung gilt der Augenblick des Zukippens als Versuchsbeginn.

Inbalt der Gefäße: Trog aller Gefäße $0,1 \mathrm{ml} 1 n \mathrm{KOH}$. Tabellenwerte in $\mathrm{ml}$.

\begin{tabular}{lcccc}
\hline & $\mathrm{I}$ & $\mathrm{II}$ & $\mathrm{III}$ & IV \\
\hline Hauptraum: & $1 \mathrm{Blut}$ & $1 \mathrm{Blut}$ & $0,6 \mathrm{Plasma}$ & $0,6 \mathrm{Plasma}$ \\
Anhang: & $0,2 \mathrm{H}_{2} \mathrm{O}$ & $0,2 \mathrm{H}_{2} \mathrm{O}$ & $0,2 \mathrm{H}_{2} \mathrm{O}$ & $0,2 \mathrm{H}_{2} \mathrm{O}$ \\
& & mit $6 \mu \mathrm{Mol}$ & & mit $6 \mu \mathrm{Mol}$ \\
& & $\mathrm{CoSO}_{4}$ & & $\mathrm{CoSO}_{4}$ \\
\hline
\end{tabular}




\section{Ergebnisse}

Das Ergebnis ist in der folgenden Abbildung graphisch dargestellt. In der Kurve sind die Mittelwerte von je 4 Versuchen eingetragen.

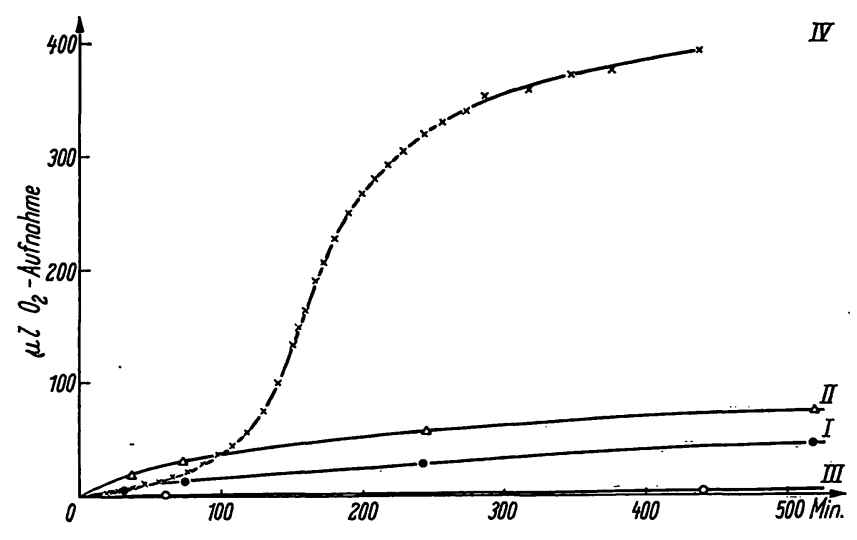

Plasma von Nüchtern-Blut und von Blut, das unmittelbar nach Nahrungszufuhr entnommen wurde, verhielt sich gleichartig. Zugabe von Glucose $+\mathrm{CoSO}_{4}$ zum Plasma (Endkonzentration 0,3\% Glucose) ergab den gleichen Effekt. Auch die $\mathrm{O}_{2}$-Aufnahme von Blut in Gegenwart und Abwesenheit von $\mathrm{Co}^{2+}$ wurde durch Glucose (Endkonzentration 0,2\%) nicht beeinflußt. Zugabe von $0,6 \mu \mathrm{Mol} \mathrm{CoSO}{ }_{4}$, gelöst in $0,2 \mathrm{~m} l$ Wasser, zu $1 \mathrm{ml}$ Blut erhöht die $\mathrm{O}_{2}$-Aufnahme kaum meßbar. Zugabe einer geringeren $\mathrm{Co}^{2+}$-Menge zu Plasma (Endkonzentration $10^{-4} \mathrm{~m}$ ) läßt auch die $\mathrm{O}_{2}$-Aufnahme von Plasma unbeeinflußt.

\section{Diskussion}

Methodik: Vor Beginn unserer Versuche bestand für uns die klinische Fragestellung der Kobaltwirkung bei Anämie. Wir haben die Stoffwechselmessungen der Blutzellen in Eigenplasma durchgeführt, da man nach WARBURG (1) in Salzlösungen nur Stoffwechsel-Artefakte mißt. Eine Isolierung der Erythrocyten allein haben wir nicht durchgeführt. Es ist uns klar, daß die Stoffwechselmessungen bei dieser Methodik nur orientierenden Charakter haben. Der Fehler ist jedoch nicht groß; denn der relativ hohen Atmung der Leucocyten und Thrombocyten $(2,3)$ steht der größere Mengenanteil der Erythrocyten gegenüber. Für wichtiger halten wir es, bei Stoffwechseluntersuchungen an Erythrocyten streng darauf zu achten, daß die Zellen immer genau zu der gleichen Zeit nach Blutentnahme zur Messung kommen und in der Zwischenzeit jeweils gleichen Bedingungen ausgesetzt sind.

Ergebnisse: Zwei Ergebnisse sind festzuhalten: die starke Sauerstoff-Aufnahme von Plasma mit 7,5 $\mathrm{mMol} \mathrm{Co}^{2+}$ und die nahezu völlige Hemmung dieser $\mathrm{O}_{2}$-Aufnahme durch Blutzellen. - Man kann daran denken, daß $\mathrm{Co}^{2+}$ mit Plasma-Bestandteilen autoxydable Schwefelverbindungen bildet, die sich zur Sulfat- bzw. SulfonsäureStufe aufoxydieren. Zur Oxydation von $6 \mu \mathrm{Mol} \mathrm{CoS} \mathrm{zu}$ $\mathrm{CoSO}_{4}$ werden jedoch nur $270 \mu l \mathrm{O}_{2}$ verbraucht, und $0,6 \mathrm{~m} l$ Plasmà enthalten nach den Angaben der Literatur größenordnungsmäßig nur $0,6 \mu \mathrm{Mol}$ Gesamt-Schwefel. Da sich das Plasma im Lauf des Versuchs in Gegenwart von $\mathrm{Co}^{2+}$ auch dunkel verfärbt, ist wohl eher an eine $\mathrm{Co}^{2+}=$ katalysierte Autoxydation von Plasma-Lipoiden zu denken. Hierfür spricht auch die Nicht-Beeinflussung der $\mathrm{O}_{2}$-Aufnahme durch Glucose. - Schwierig ist auch die Hemmung der beschriebenen Autoxydation durch Blutzellen zu erklären. Wenn das zugefügte $\mathrm{Co}^{2+}$ vollständig von den roten Blutzellen aufgenommen würde, kämen auf $3 \mathrm{Fe}$ der Erythrocyten immerhin 2 Co. Man sollte trotzdem die Permeabilität der ErythrocytenMembran für $\mathrm{Co}^{2}+$ bei unserer Versuchsanordnung und die Bindungsfähigkeit von Kobalt im Erythrocyten untersuchen, um erkennen zu können, ob möglicherweise bei der beschriebenen Hemmwirkung Vorgänge an der Oberfläche der Erythrocyten entscheidend sind. Eine dritte Erklärungsmöglichkeit wäre die, daß bei den beobachteten Autoxydationen intermediär $\mathrm{H}_{2} \mathrm{O}_{2}$ entsteht und dieses infolge der hohen Katalase-Aktivität der Erythrocyten zersetzt wird, in reinem Plasma jedoch größtenteils unzersetzt bleibt. In diesem Fall läge keine Hemmwirkung der Blutzellen auf die Autoxydation des Plasmas vor.

Eine wesentliche Konsequenz der Versuchsergebnisse besteht darin, bei allen Stoffwechseluntersuchungen mit Kobalt - und wohl auch mit anderen Schwermetallen streng darauf $\mathrm{zu}$ achten, ob nicht die benutzte Inkubationsflüssigkeit allein mit den Metallen Wechselwirkungen eingeht, die in Gegenwart von Zellen nicht beobachtet werden. Bei Nichtbeachtung dieser Maßnahme sind falsche Interpretationen der Einwirkungen vor. Metallionen auf den Zellstoffwechsel möglich.

Frl. W. Schwartz ist für Blutentnahmen und sorgfältige Hilfe beim Ablesen der Manometer zu danken.

\section{Literatur}

1. Warburg, O., K. Gawehn und A.-W. Geissler, Z. Natur-' forsch. 12b, 115 (1957). - 2. WARBnRG, O., K. GAWEHN und A.-W. Gerssler, Z. Naturforsch. 13b, 515 (1958). - 3. KRebs,
Sir H. A., R. B. Clayton, H. L. Kornaerg, J. M. Lowenstein und J. R. Quayle, „Documenta Geigy“, 6. Aufl., S. 365, Basel (1960).
Dr. rer. nat. Jürgen Dittmann Laboratorium der Universitäts-Kinderklinik 665 Homburg (Saar) 